\title{
APPLICATIONS OF SOFT COMPUTIONG IN ENGINEERING PROBLEMS
}

\author{
Hitoshi Furuta, Koichiro Nakatsu and Hiroshi Hattori \\ *Department of Informatics, Kansai University, Takatsuki, Osaka 569-1095, \\ Japan \\ furuta@res.kutc.kansai-u.ac.jp, inside2@sc.kutc.kansai-u.ac.jp, \\ hattori@sc.kutc.kansai-u.ac.jp
}

Keywords: Artificial Intelligence, Adaboost, Damage Detection, Fuzzy Logic, Genetic Algorithm, GMDH, Life-Cycle Cost, Maintenance Program, Restoration Scheduling.

\section{Introduction}

Recently, great attention has been paid to soft computing technology, because of its applicability and easiness of computation in engineering problems. This chapter introduces several applications of the soft computing in various real engineering problems. First, a new optimal restoration scheduling method is described, which was developed for damaged road networks by using Genetic Algorithm (GA). The method can propose an early restoration plan for lifeline systems after earthquake disasters. Here, two issues are focused on, the first of which is such an allocation problem that which groups will restore which disaster places, and the second is such a scheduling problem what order is the best for the restoration. In order to solve the two problems simultaneously, GA is applied, because it has been proven to be very powerful in solving combinatorial problems. However, road networks after earthquake disasters have an uncertain environment, that is, the actual restoring process should be performed by considering various uncertainties simultaneously. Therefore, GA Considering Uncertainty (GACU) was developed to treat various uncertainties involved.

Next, an optimal maintenance planning of bridge structures using multi-objective genetic algorithm is described, which can provide several practical scheduling candidates that the bridge owner can select by considering the situation and constraints.

A structural health monitoring system is introduced, which can treat the changes of systems and environments. By adapting to the environment, it is not necessary to prepare any previous knowledge and examination for the underlying structures and environment. In other words, it is not necessary to use a precise modelling and analysis method before conducting the health monitoring. In the system, both Adaboost and GMDH (Group Method of Data Handling) are used for the learning and compared by paying attention to the accuracy of prediction. 
In order to establish a rational maintenance program for structures, it is necessary to collect enough data about the material and structural characteristics and to evaluate the structural damage in a quantitative manner. However, it is difficult to avoid the subjectivity of inspectors when visual data are used for the evaluation of damage or deterioration. The method can evaluate the damage condition of existing structures by using the visual information given by digital photos. It is based upon such new technologies as image processing, photo-grammetry, pattern recognition, and artificial intelligence.

\section{Optimal Restoration Scheduling of Damaged Road Networks Using Genetic Algorithm}

The purpose of this research is to propose an early restoration for lifeline systems after earthquake disasters. Here, two issues are focused on, the first of which is such an allocation problem that which groups will restore which disaster places, and the second is such a scheduling problem what order is the best for the restoration. In order to solve the two problems simultaneously, Genetic Algorithm (GA) is applied, because it has been proven to be very powerful in solving combinatorial problems. However, road networks after earthquake disasters have an uncertain environment, that is, the actual restoring process should be performed by considering various uncertainties simultaneously. GA Considering Uncertainty (GACU) can treat various uncertainties involved, but it is difficult to obtain the schedule which has robustness. In this study, an attempt is made to develop a decision support system of the optimal restoration scheduling by using the improved GACU.

\subsection{Genetic Algorithm Considering Uncertainty}

Here, it is assumed that a road network is damaged, in which multiple portions are suffered from damage so that it cannot function well. The objective of this study is the realization of quick restoration of the lifeline system. It is intended to determine the optimal allocation of restoring teams and optimal scheduling of restoring process. Then, the following conditions should be taken into account [1] [2]:

1. The optimal allocation of restoring team, optimal scheduling of restoring process, and optimal selection of restoring method must be determined simultaneously.

2. A portion of the road network is suffered from several kinds of damage that have a hierarchical relation in time.

As an example of restoration, a road network is considered, which has 164 nodes as shown in Figure 1. This model corresponds to an area damaged by the 1995 Kobe earthquake. For this road network, the following restoration works are necessary to recover the function:

1. work (A): work to clear the interrupted things : 38 sites $(1-38)$

2. work (B) : work to restore the roads : 50 sites $(1-50)$

Then, the limitation and restriction of each work should be considered, for instance, work (B) should be done after work (A). Work (B) consists of the following three works; work to repair the roads, work to reinforce the roads and work to rebuild the roads. The waiting places of restoring teams for work (A) and work (B) are shown by the number A (1-8) and B $(1-8)$, respectively. 


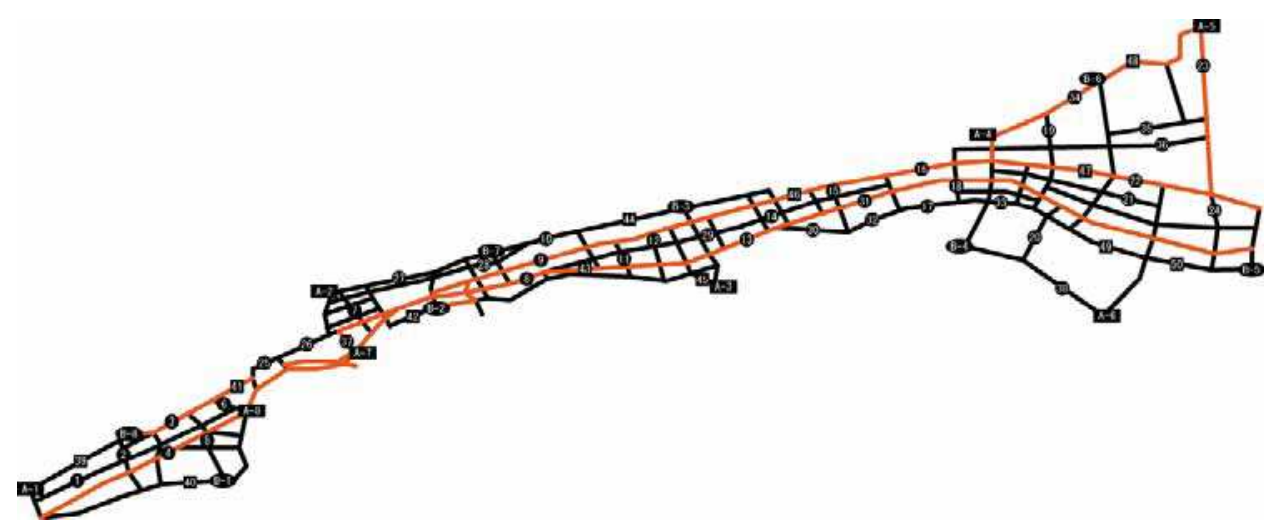

Fig. 1. Road network model

\subsection{Restoration Scheduling}

Weighting factors are prescribed for the links with damage, which are denoted by $w_{i}$ $\left(i=1 \sim n_{L}\right) . n_{L}$ is the total number of damaged links. Then, the restoring rate after $q$ days, $R q$, is expressed as follows:

$$
R^{q}=\frac{\sum_{i \in J^{q}} w_{i} \times l_{i}}{\sum_{i \in J^{\circ}} w_{i} \times l_{i}}
$$

where $l_{i}$ is the distance of the $i$-th link, $J^{0}$ is the set of damaged links, $J q$ is the number of restored links until $q$ days after the disaster, and $w_{i}$ is the weighting factor of the $i$-th link. Then, the objective function can be calculated by using the restoring day and the restoring rate.

Restoring times are calculated for each restoring work, and the minimum days necessary for each work is given as

$$
d=h / t_{1}
$$

where $h$ is the restoration time required to complete the restoration work. In this research, the restoration time is calculated by using the restoration rate for each work and the capability value. The relation between the restoration rate for each work and the capability of the teams are shown in Figure 2. The restoration rate is given as follows:

a) Small damage: In the small damage, there is no difference in capability between each team. The restoration will be completed during a fixed time. Here, 4 hours are assumed.

$$
h=h_{t}
$$

b) Moderate damage: In the moderate damage, there is some difference in capability between every teams, however, every teams can restore the damage.

$$
h=D / A
$$


where $D$ is the amount of damage and $A$ is the capability of the team, that is, the restoring amount per an hour.

c) Large damage: In the large damage, only some teams can restore, because other teams have no restoring equipment and facility necessary for the large damage.

$$
\begin{aligned}
& h=\infty\left(A<A_{c}\right) \\
& h=D / A\left(A \geq A_{c}\right)
\end{aligned}
$$

The working hours per day of a restoration team is calculated by Equation 6, where $t_{m}$ is the moving time to a site given by Equation 7. The shortest distance from the waiting place of the restoration team to the site is expressed as $L(\mathrm{~km})$, and the moving speed of the team is set to be $v(\mathrm{~km} / \mathrm{h}) . h_{c}$ is the preparation time that is necessary for every works.

$$
\begin{gathered}
t_{1}=t_{0}-2 t_{m}-h_{c} \\
t_{m}=L / v
\end{gathered}
$$

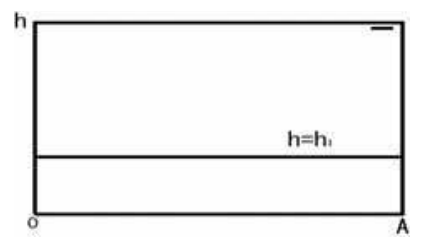

a) Small damage

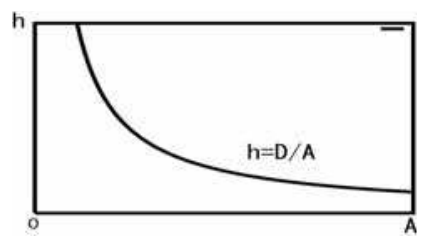

b) Moderate damage

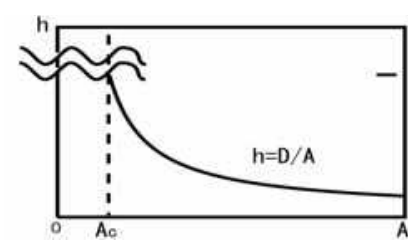

c) Large damage

Fig. 2. Relations between restoration rate for each work and capability of teams

\subsection{Influence of Uncertainty}

At a devastated area after an earthquake disaster, the circumstances are changing with aftershock, fire and bad weather. The devastated area may have another damaged and the circumstances may not be constant. This is due to the uncertainty of a damage which occurs from the followings:

1. Delay: Delay induces the increase of restoring days of a work. The delay of the work influences the whole restoring schedule.

2. Impossibility to restore: Impossibility to restore is the situation that a team without sufficient restoring equipment and facility is assigned to large damage work. Such a team cannot restore the large damage work. Impossibility of work to restore causes failure of restoring schedule.

\subsection{Genetic Algorithm Considering Uncertainty}

In order to obtain the restoration schedule which has robustness to the uncertainty of damage, it is necessary to implement sampling many times. In GA considering uncertainty (GACU) [3], objective function is defined as the expected value of $F^{\prime}(x)$ to consider the search process as the sampling. 


$$
F^{\prime}(x)=F(x) \text { with Uncertainty }
$$

$F(x)$ contains a variable element, that is, uncertainty, so that $F^{\prime}(x)$ is changing according to the uncertainty. It is assumed that the number of sampling is age of individual. This sampling is performed by considering the evolution mechanism of inheritance, that is, gene of parents is resembled to that of children. The procedure of GACU is given as follows:

STEP 1. Generation of initial population

STEP 2. Selection of parents

STEP 3. Crossover and mutation: generation of new individuals

STEP 4. Evaluation: evaluation of new individuals and re-evaluation and adding age of alive individuals

STEP 5. Natural selection

STEP 2 to 5 are repeated until the convergence is achieved

\subsection{Uncertainty of Optimal Restoration Schedule}

When a restoration team arrives at the disaster site, the disaster circumstances may be different from those predicted, because devastated situations are constantly changing by the aftershock, fire disaster and bad weather, which are likely to make damage worse. Such a change of devastated area affects the scheduling process, because it takes more days than those scheduled, and furthermore it may be impossible to restore unless the restoration teams have enough ability. Therefore, in this paper, the amount of damage and the delay will be treated as uncertain factors and the restoration scheduling problem is formulated as an optimization problem with uncertainties. The influences of delay are shown in Figure 3.

Fig. 3. The influences of delay

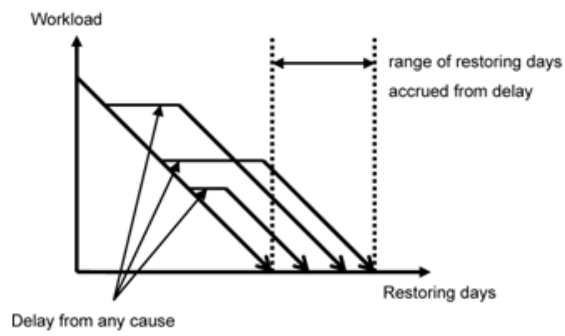

\subsection{Application of Genetic Algorithm Considering Uncertainty}

GACU is applied to obtain the optimal robust restoration schedule. Table 1 presents the parameters of GACU used here. The optimal robust schedule is presented in Figures 4 and 5. The effects of increasing the damage examined by 1000 simulations are shown in Table 2 . Table 3 presents the effects of the delay examined by 1000 simulations. It is seen that teams without restoring equipment are not assigned to large damage works and medium damage works which are changeable to large damage and waiting time is properly assured to avoid the effects of delay. In addition, most of larger damage works are assigned to restoration team with high ability. The schedule is not only robust but also optimum for the early restoring. In this paper, assuming that a road network has an uncertain damage, it is intended to obtain the optimal restoring schedule considering uncertainty. From the results 
obtained, it is concluded that the proposed method using GACU is useful for obtaining the optimal restoring schedule with robustness to uncertainty of damage.

\begin{tabular}{c|c|c|c}
\hline Population & $\begin{array}{c}\text { Probability of } \\
\text { Crossover }\end{array}$ & $\begin{array}{c}\text { Probability of } \\
\text { Mutation }\end{array}$ & Generation \\
\hline 500 & 0.6 & 0.005 & 2000 \\
\hline
\end{tabular}

Table 1. Parameters of GACU

\begin{tabular}{|c|c|c|c|c|c|c|c|c|c|}
\hline Days & \begin{tabular}{l|l}
12 \\
\end{tabular} & \begin{tabular}{l|l}
3 \\
\end{tabular} & \begin{tabular}{l|l}
45 & 5
\end{tabular} & 6 & 7 & \begin{tabular}{|l|}
8 \\
\end{tabular} & \begin{tabular}{l|l}
9 & 11 \\
\end{tabular} & 911 & \begin{tabular}{|l|l|}
13 & 13 \\
\end{tabular} \\
\hline Groupl & \multicolumn{3}{|c|}{13} & \multicolumn{4}{|c|}{2} & \multirow{2}{*}{\multicolumn{2}{|c|}{ 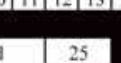 }} \\
\hline 2 & 34 & 33 & & 35 & & & 31 & & \\
\hline 3 & 17 & & 14 & & 10 & & 12 & & \\
\hline 4 & 37 & & 88 & & & 5 & & & 24 \\
\hline 5 & 4 & & & 22 & & 32 & 21 & & 28 \\
\hline 6 & \begin{tabular}{|l|l|}
36 \\
\end{tabular} & 11 & & 18 & & 1 & & 26 & \\
\hline 7 & 20 & \begin{tabular}{|l|}
23 \\
\end{tabular} & 16 & & 6 & & 8 & & 30 \\
\hline 8 & 3 & $7 \mid$ & 1 & & 9 & & 29 & & 19 \\
\hline
\end{tabular}

Fig. 4. The optimal robust schedule of Work (A)

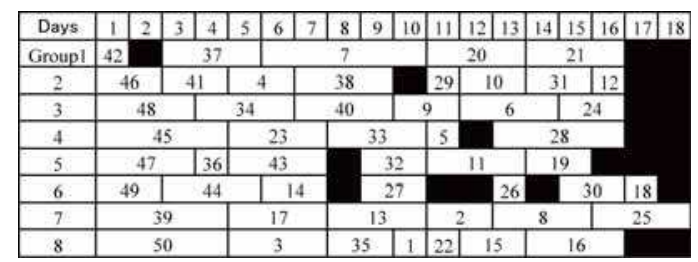

Fig. 5. The optimal robust schedule of Work (B)

\begin{tabular}{c|c|c}
\hline Probability changed & Average of evaluation & Impossible to restore \\
\hline $5 \%$ & 6.87 & $0 / 1000$ \\
\hline $10 \%$ & 7.00 & $0 / 1000$ \\
\hline $20 \%$ & 7.27 & $0 / 1000$ \\
\hline
\end{tabular}

Table 2. Effects of increasing the damage examined by 1000 simulations

\begin{tabular}{c|c|c|c}
\hline $\begin{array}{c}\text { Probability } \\
\text { changed }\end{array}$ & Evaluation & SimpleGA & GACU \\
\hline \hline \multirow{5}{*}{$5 \%$} & Evaluation(Ave) & 7.52 & 7.88 \\
\cline { 2 - 4 } & Evaluation(Max) & 8.80 & 7.97 \\
\cline { 2 - 4 } & Evaluation(Min) & 7.11 & 7.18 \\
\cline { 2 - 4 } & Standard deviation & 0.52 & 0.47 \\
\hline \hline \multirow{5}{*}{$10 \%$} & Evaluation(Ave) & 9.12 & 7.91 \\
\cline { 2 - 4 } & Evaluation(Max) & 17.22 & 8.31 \\
\cline { 2 - 4 } & Evaluation(Min) & 7.11 & 0.18 \\
\cline { 2 - 4 } & Standard deviation & 2.12 & 8.01 \\
\hline \hline \multirow{5}{*}{$20 \%$} & Evaluation(Ave) & 15.04 & 8.41 \\
\cline { 2 - 4 } & Evaluation(Max) & 17.56 & 7.20 \\
\cline { 2 - 4 } & Evaluation(Min) & 14.35 & 1.29 \\
\cline { 2 - 4 } & Standard deviation & 3.22 & \\
\hline \hline
\end{tabular}

Table 3. Effects of the delay examined by 1000 simulation

\section{Optimal Maintenance Planning of Bridge Structures Using Multi-Objective Genetic Algorithm}

In order to establish a rational maintenance program, it is necessary to develop a costeffective decision-support system that can provide us with a practical and economical plan 
[4]. Although low-cost maintenance plans are desirable for bridge owner, it is necessary to consider various constraints when choosing an appropriate actual maintenance program. For example, the minimization of maintenance cost requires to prescribe the target safety level and the expected service life time. The predetermination of requirements may lose the variety of possible maintenance plans. Namely, it may be possible to find out a better solution that can largely extend the service life if the safety level can be sensitively decreased even with the same amount of maintenance cost.

\subsection{Concrete Bridge Model}

A group of ten concrete highway bridges are considered in this study. Maintenance management planning for ten consecutive piers and floor slabs (composite structure of steel girders and reinforced concrete (RC) slabs) is considered here [5]. Each bridge has the same structure and is composed of six main structural components: upper part of pier, lower part of pier, shoe, girder, bearing section of floor slab, and central section of floor slab.

Environmental conditions can significantly affect the degree of deterioration of the structures and may vary from location to location according to geographical characteristics such as wind direction, amount of splash, etc. To take the environmental conditions into account, the deterioration type and year from completion of each bridge are summarized in Table 4.

\begin{tabular}{c|c|c}
\hline $\begin{array}{c}\text { Bridge } \\
\text { number }\end{array}$ & $\begin{array}{c}\text { Years from } \\
\text { completion }\end{array}$ & Deterioration type \\
\hline \hline B01 & 2 & neutralization of concrete \\
\hline B02 & 2 & neutralization of concrete \\
\hline B03 & 0 & chloride attack (slight) \\
\hline B04 & 0 & chloride attack (medium) \\
\hline B05 & 0 & chloride attack (severe) \\
\hline B06 & 0 & chloride attack (medium) \\
\hline B07 & 0 & chloride attack (severe) \\
\hline B08 & 1 & chloride attack (medium) \\
\hline B09 & 1 & chloride attack (slight) \\
\hline B10 & 1 & chloride attack (slight) \\
\hline
\end{tabular}

Table 4. Years from completion and type of deterioration caused by environmental conditions

\subsection{Maintenance Strategies and Life-Cycle Cost}

In order to prevent deterioration in structural performance, several options such as repair, restoring, and reconstruction are considered. Since the effects may differ even under the same conditions, average results are adopted here. Maintenance methods applicable to RC slab may vary according to the environmental conditions and are determined considering several assumptions [6].

Life-Cycle Cost (LCC) is defined as the total maintenance cost for the entire bridge group during its life. This is obtained by the summation of the annual maintenance costs through the service life of all the bridges. The future costs are discounted to their present values. However, the discount rate is assumed to be zero in this study. Other costs, such as indirect 
construction costs, general costs, and administrative costs, etc., are calculated in accordance with Cost Estimation Standards for Civil Construction [7]. The direct construction costs consist of material and labor costs and the cost of scaffold. For calculating the construction costs, the following assumptions are taken into account:

1. The cost of scaffold can be reduced by sharing. For example, scaffold can be shared for repairing the bearing and the bearing section of $\mathrm{RC}$ slab, consequently reducing the scaffolding cost.

2. Indirect construction costs, such as general administrative costs, can be saved by implementing several repairs in the same year. The ratio of indirect to maintenance costs decreases as the direct costs increase. The value of LCC is reduced when multiple components are repaired simultaneously.

\subsection{Multi-Objective Genetic Algorithm (MOGA)}

Genetic Algorithm (GA) is an evolutionary computing technique, in which candidates of solutions are mapped into GA space by encoding. The following steps are employed to obtain the optimal solutions [8]: a) initialization, b) crossover, c) mutation, d) natural selection, and e) reproduction. Individuals, which are solution candidates, are initially generated at random. Then, steps b, c, d, and e are repeatedly implemented until the termination condition is fulfilled. Each individual has a fitness value to the environment. The environment corresponds to the problem space and the fitness value corresponds to the evaluation value of objective function. Each individual has two aspects: Gene Type (GTYPE) expressing the chromosome or DNA and Phenomenon Type (PTYPE) expressing the solution. GA operations are applied to GTYPE and generate new children from parents (individuals) by effective searches in the problem space, and extend the search space by mutation to enhance the possibility of individuals other than the neighbour of the solution. GA operations that generate useful children from their parents are performed by crossover operation of chromosome or genes (GTYPE) without using special knowledge and intelligence. This characteristic is considered as one of the reasons of the successful applications of GA.

\subsection{Application of MOGA to Maintenance Planning}

It is desirable to determine an appropriate life-cycle maintenance plan by comparing several solutions for various conditions [6]. A new decision support system is developed here from the viewpoint of multi-objective optimization, in order to provide various solutions needed for the decision-making.

In this study, LCC, safety level and service life are used as objective functions. LCC is minimized, safety level is maximized, and service life is maximized. There are trade-off relations among the three objective functions. For example, LCC increases when service life is extended, and safety level and service life decrease due to the reduction of LCC. Then, multi-objective optimization can provide a set of Pareto solutions that cannot improve an objective function without making other objective functions worse.

Then, objective functions are defined as follows: 


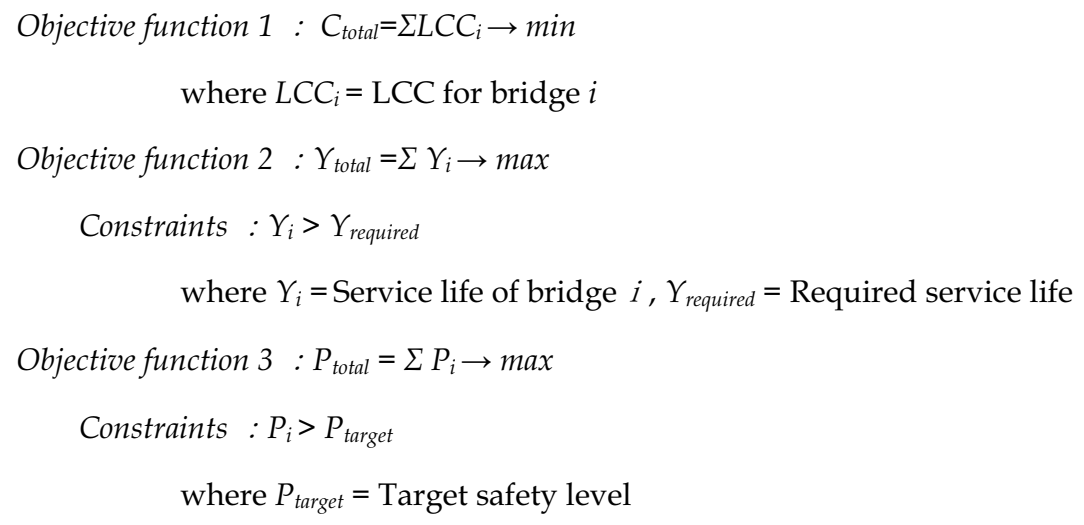

The above objective functions have trade-off relations to each other. Namely, the maximization of safety level or maximization of service life cannot be realized without increasing LCC. On the other hand, the minimization of LCC can be possible only if the service life and/or the safety level decreases.

\subsection{Numerical Example}

In the implementation of MOGA, the GA parameters considered are as follows: number of individuals $=2000$, crossover rate $=0.60$, mutation rate $=0.05$ and number of generations $=$ 5000. Figures 6 to 9 present the results obtained by MOGA. Each figure shows the comparison of the results of the 1st generation (iteration number) and the 5000th generation. In Figure 6, the solutions at the 1st generation spread over the design space. This means that the initial solutions can be generated uniformly. After the 5000th generation, the solutions tend to converge to a surface, which finally forms the Pareto set as the envelope of all solutions. The number of solutions at the 5000th generation is much larger than that at the 1st generation. This indicates that MOGA could obtain various optimal solutions with different LCC values, safety levels, and service lives. From Figure 6, it is seen that MOGA can find out good solutions, all of which evolve for all the objective functions, and the final solutions are sparse and have discontinuity. In other words, the surfaces associated with the trade-off relations are not smooth. This implies that an appropriate long term maintenance plan cannot be created by the repetition of the short term plans.

In Figure 7, the vertical axis represents safety level, whereas the horizontal axis represents LCC. Although at the 1st generation, the solutions may have a rather linear relation between safety level and LCC, the relation shows non-linearity through the convergence process. This implies that the safety level may be significantly increased if the LCC can be slightly increased, when the service life is fixed. Figure 8 presents the relation between LCC and service life. Since LCC and service life have a rather perfect positive linear correlation, it can be said that the service life can be extended if LCC can be increased. On the other hand, there is no distinct relation between safety level and service life, as shown in Figure 9. It should be noted that the safety level may not be raised even if the service life is shorten, under a constant LCC. Namely, the relation between safety level and service life is so unclear that the extension of service life should be done with careful examination. 
Finally, it is confirmed that the proposed method using MOGA can provide many nearoptimal maintenance plans with various reasonable LCC values, safety levels and service lives.

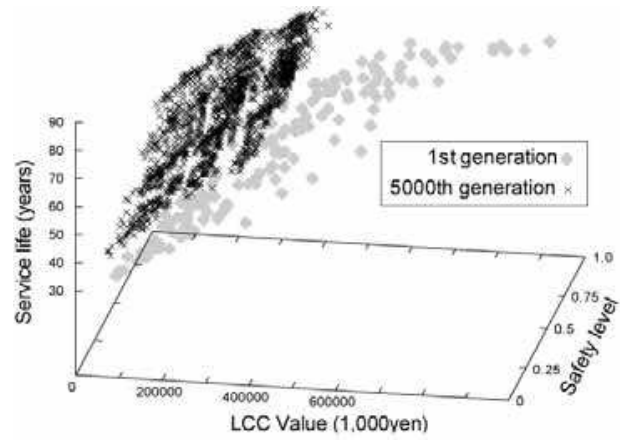

Fig. 6. Pareto solutions obtained by MOGA

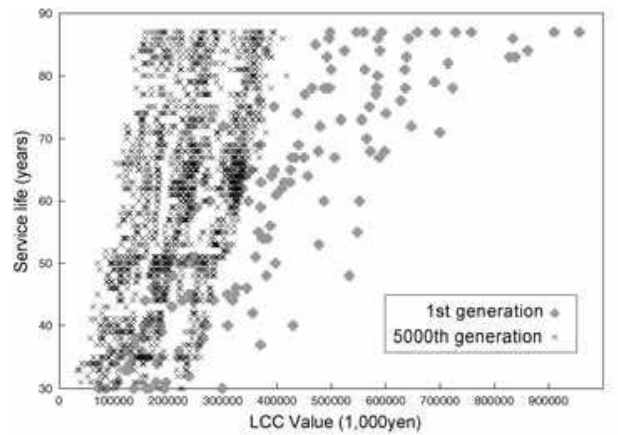

Fig. 8. Relation between LCC and service life

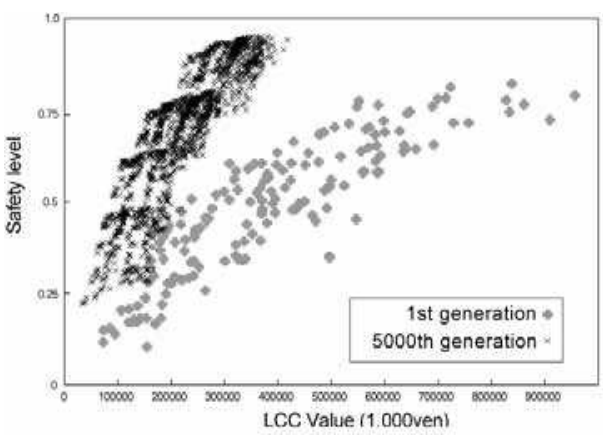

Fig. 7. Relation between LCC and safety level

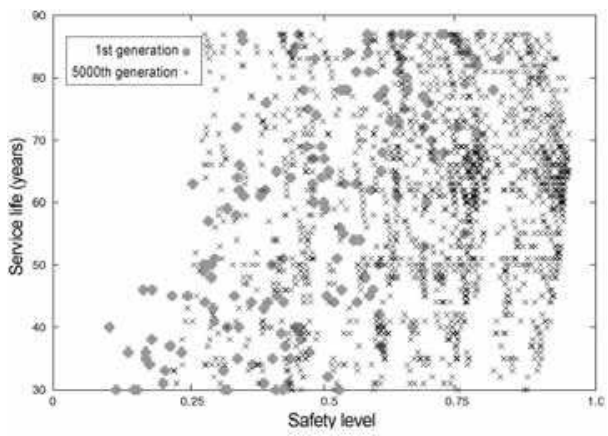

Fig. 9. Relation between safety level and service life

\section{Health Monitoring System Using Learning System}

Recently, many researches have been made on health monitoring of existing structures such as buildings, bridge and other civil structures. Many structures are becoming superannuated and deteriorated. Furthermore, in Japan, natural disasters like typhoon and earthquake have occurred frequently so that the damage assessment of existing structures is very important. In order to evaluate the damage state of structures health monitoring technology is quite promising to provide useful information. In the health monitoring, there are still some problems in modelling, analysis and experimental examination for practical use.

An attempt was made to develop a structural health monitoring system that can adapt to the structural systems and environments, by introducing the learning ability. By introducing the learning ability, it is not necessary to prepare any previous knowledge and examination for the underlying structures and environment. In other words, it is not necessary to use the precise modelling and analysis method before conducting the health monitoring. 


\subsection{AdaBoost}

Boosting method uses such two learning algorithm with high precision (Strong learning algorithms) and learning algorithm with low precision (Weak learning algorithm). AdaBoost is one of the Boosting methods. AdaBoost is used for pattern recognition problems frequently.

The AdaBoost creates several learning hypotheses by using given weak learning algorithms at a round cycle. At the round cycle, re-sampling of learning data are performed by using the given probability distribution. At the next round, probability is updated to choose data that make errors at the round. By repeating this process, it is possible to obtain plural hypothesis that have different characteristics. The strong algorithm gives unification by combining each weak learning algorithm with weights. Figure 10 shows the concept of AdaBoost.

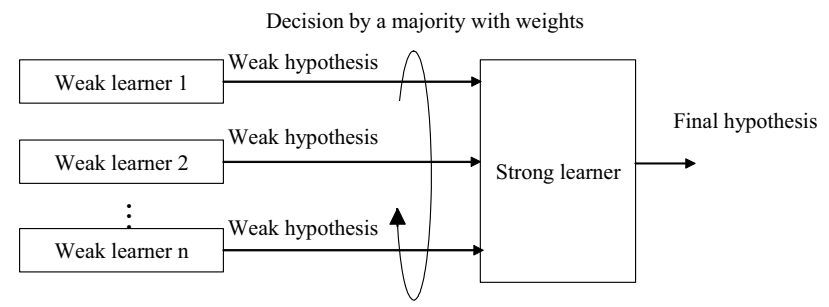

Fig. 10. Conception of AdaBoost

AdaBoost executes the recognition by combining plural weak learning algorithms like Figure 10, where no restriction exists on each weak learning algorithm. For even equal number of input and output data, it allows to use all different algorithms. By using a soft computing method like neural network for the weak learning algorithm, it is possible to obtain the same advantage.

The AdaBoost can obtain the high quality and versatile hypothesis by few teaching data. It also has such merits that algorithm is easy and the number of parameters adjusted is few.

\subsubsection{Procedure of Adaboost}

Adaboost can apply to the pattern recognition problem with multiple classifications. In this case, the 2 -value with -1 and 1 is treated. Adaboost is repeated $t(t=1,2,3, \ldots, T)$ times. Procedure of Adaboost is shown below.

Step 1: Obtain the learning data

Give the teaching data $\left(\mathrm{x}_{1}, \mathrm{y}_{1}\right), \ldots \ldots . .,\left(\mathrm{x}_{\mathrm{n}}, \mathrm{y}_{\mathrm{n}}\right)$.

Step 2: Initialize the probability distribution

Initialize the probability distribution using the next equation. In this equation, $\mathrm{D}_{\mathrm{t}}(\mathrm{i})$ is the probability distribution of teaching data $\mathrm{i}$ at round $\mathrm{t}$.

$$
\mathrm{D}_{\mathrm{t}}(\mathrm{i})=1 / \mathrm{m}
$$

Step 3: Steps 4 to 6 as a round and repeat $\mathrm{T}$ times 
Step 4: Obtain weak hypothesis $h_{t}$

By learning setting times of weak learning algorithm, obtain weak hypothesis. The learning data is chosen by probability based $D_{t}(i)$. Then, precision of weak hypothesis is calculated by the next equation using probability of error using $\mathrm{D}_{\mathrm{t}}$.

$$
\varepsilon_{t}=P_{r i-D i}\left[h_{t}\left(x_{i}\right) \neq y_{i}\right]=\sum_{i: h_{t}\left(x_{i}\right) \neq y_{i}} D_{t}(i)
$$

Step 5: Decide the importance of weak hypothesis

Decide the importance of weak hypothesis used the majority decision with the weight by the next equation.

$$
\alpha_{t}=\frac{1}{2} \ln \left(\frac{1-\varepsilon_{t}}{\varepsilon_{t}}\right)
$$

Step 6: Update probability distribution

Update probability distribution of teaching data by the recognition result when using weak hypothesis $\mathrm{h}_{\mathrm{t}}$. Probability of teaching data producing the error recognition by $h_{t}$ is increased, and learning is concentrated to the difficult teaching data. Equation to update of the probability distribution is as follows:

$$
\begin{aligned}
D_{t+1}(i) & =\frac{D_{t}(i)}{Z_{t}} \times\left\{\begin{array}{l}
e^{-a t}\left(h_{t}\left(x_{i}\right)=y_{i}\right) \\
e^{a t}\left(h_{t}\left(x_{i}\right) \neq y_{i}\right)
\end{array}\right. \\
& =\frac{D_{t}(i) e^{-\alpha_{t} y_{i} h_{t}\left(x_{i}\right)}}{Z_{t}}
\end{aligned}
$$

In this equation, $Z_{t}$ is the factor to normalize the probability distribution.

Step 7: Obtain final hypothesis

Final hypothesis $\mathrm{H}_{t}$ is obtained by the majority decision with weighs of weak hypotheses. The equation to obtain the $\mathrm{H}_{t}$ is .

$$
H(x)=\operatorname{sgn}\left(\sum_{t=1}^{T} \alpha_{t} h_{t}(x)\right)
$$

\subsection{Vibration Analysis}

In this section, vibration analysis is done by numerical simulation. At the numerical simulation, deterioration of objective structure and the vibration characteristics of structural change are assumed. Vibration at intact situation and deteriorated situation are compared using a multiple freedom structure. Through the numerical simulation, it is concluded that the method can find the deterioration of structure by analyzing vibration response.

\subsubsection{Vibration}

Vibration of structure can be defined by the next equation. 


$$
M \cdot u^{\prime \prime}(t)+C \cdot u^{\prime}(t)+K \cdot u(t)=0
$$

In the equation, $\mathrm{u}(\mathrm{t})$ is the displacement of structure at time $\mathrm{t}, \mathrm{M}$ is mass, $\mathrm{C}$ is damping ratio and $\mathrm{K}$ is stiffness. Equation 17 has no external force, and therefore it is called free vibration. However, usually external force exists.

$$
M \cdot u^{\prime \prime}(t)+C \cdot u^{\prime}(t)+K \cdot u(t)=p(t)
$$

where $p(t)$ is external force.

\subsubsection{Wind force}

In this research, wind is used for the external force. Wind force is calculated by wind velocity as follows:

$$
F=\frac{1}{2} C \rho A V^{2} \sin ^{2} \alpha
$$

where $C$ is wind coefficient, $\rho$ is air density, A is effective area and $\alpha$ is effect angle. Those values used in this research are shown in Table 5.

Table. 5 Parameters

\begin{tabular}{|l|l|}
\hline air density & $0.125\left(\mathrm{kgf} \cdot \mathrm{sec}^{2} / \mathrm{m}^{4}\right)$ \\
\hline Coefficient & 2 \\
\hline effect angle & $90\left({ }^{\circ}\right)$ \\
\hline effect area & $200\left(\mathrm{~m}^{2}\right)$ \\
\hline
\end{tabular}

\subsubsection{Method of vibration analysis}

Runge-Kutta method is used to solve the differential equations for the numerical simulation. The following simultaneous equations are solved.

$$
\left\{\begin{array}{l}
P=u^{\prime}(t) \\
P^{\prime}=-(C \cdot P+K \cdot u(t)+P(t)) / M
\end{array}\right.
$$

\subsubsection{Model of structure}

A three-degree-of-freedom structure is employed for the object model. Therefore, Equation 18 is extended to a matrix form.

$$
[M] \cdot\left\{u^{\prime \prime}(t)\right\}+[C] \cdot\left\{u^{\prime}(t)\right\}+[K] \cdot\{u(t)\}=\{p(t)\}
$$

In this equation, $[\mathrm{M}],[\mathrm{C}]$ and $[\mathrm{K}]$ are the matrices of mass, damping and stiffness. The matrices of three-degree-freedom structure used in this research are shown as follows: 


$$
\begin{gathered}
M=\left[\begin{array}{ccc}
m_{1} & 0 & 0 \\
0 & m_{2} & 0 \\
0 & 0 & m_{3}
\end{array}\right] \\
C=\left[\begin{array}{ccc}
c_{1}+c_{2} & -c_{2} & 0 \\
-c_{2} & c_{2}+c_{3} & -c_{3} \\
0 & -c_{3} & c_{3}
\end{array}\right] \\
K=\left[\begin{array}{ccc}
k_{1}+k_{2} & -k_{2} & 0 \\
-k_{2} & k_{2}+k_{3} & -k_{3} \\
0 & -k_{3} & k_{3}
\end{array}\right]
\end{gathered}
$$

where $\mathrm{m}_{\mathrm{i}}, \mathrm{k}_{\mathrm{i}}$ and $\mathrm{c}_{\mathrm{i}}$ are mass, damping factor and stiffness of each story.

\subsubsection{Pilot study}

In this section, numeric simulation is done using sine curve for the external force. In the numerical simulation, the intact situation and deteriorated situation at each story are compared. The deterioration is assumed to reduce $10 \%$ of stiffness at every 10000 steps. Figure 11 to Figure 13 show the difference of displacement for the intact situation and the situation that deterioration occur at each story. Figure 12 shows the difference of displacement for the intact situation and the situation that deterioration occurs at the first story.

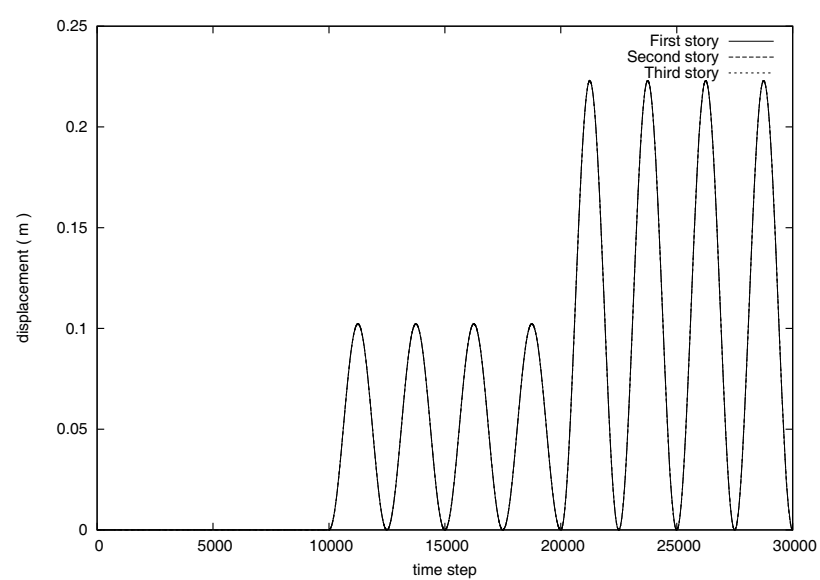

Fig. 11. Difference of displacement (Case of degradation accrual first story) 


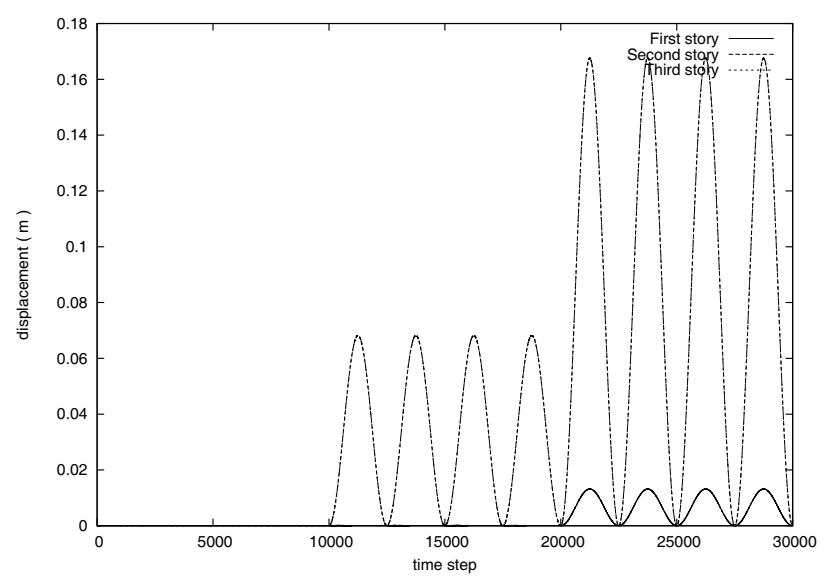

Fig. 12. Difference of displacement (Case of degradation accrual second story)

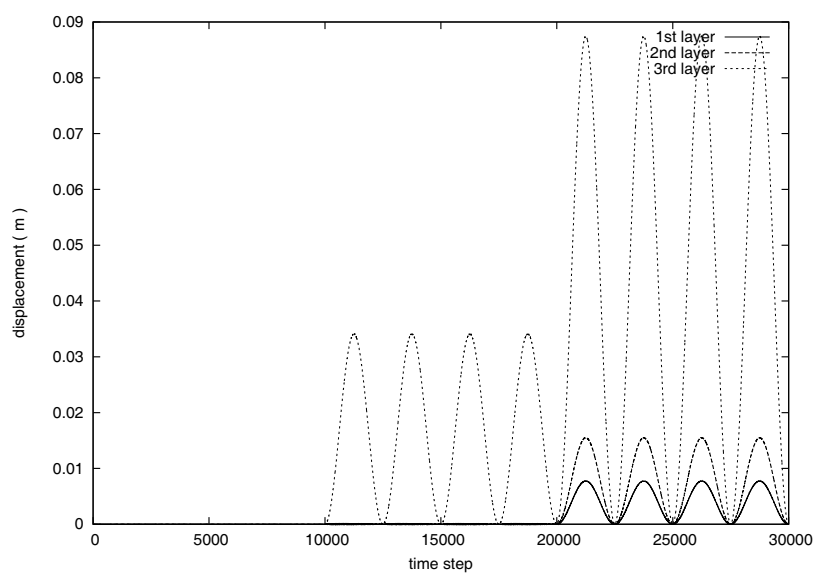

Fig. 13. Difference of displacement (Case of degradation accrual third story)

From Figure 11 to Figure 13, the behavior of structure changes by position, even the same size deterioration. Whereas the differences of response of each story is the same when the first story has deterioration, the difference of response of the first story is smaller than other stories and second story's one and third story's one are the same, when the second story has deterioration. Also, the difference of response of the third story is bigger than other stories and the first story and those of the second story is the same, when the second story has deterioration. Then, it is confirmed that it is possible to identify the difference of intact structure and the structure with damage at the i-th story. Herewith, it is possible to identify the position of deterioration by comparing the difference of response.

\subsection{Proposed System}

Structure of the proposed system is shown in Figure 14. 


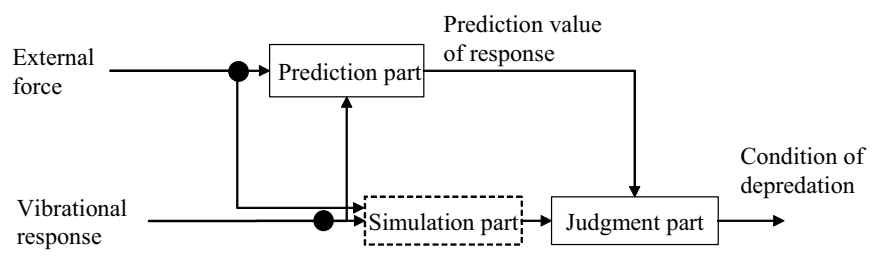

Fig. 14. Structure of proposed system

The proposed system is composed of a prediction part that learns the vibration response and predicts the next response, judgment part that detects the deterioration by analyzing the vibration response, and simulation part that analyze a vibration. Inputs to the proposed system are external force and vibration response, that is, displacement and velocity of structures. Outputs from the proposed system are the probability of deterioration and the position of deterioration.

In this research, GMDH is used for the prediction part, so that versatile rules are obtained and calculation time can be reduced. At the judgment part, fuzzy reasoning is used to detect the deterioration by comparing the prediction value and the observed value. Input data for fuzzy reasoning are prediction errors and prediction error rates. Fuzzy rule using in this research is shown in Table 6.

\begin{tabular}{|c|c||c|}
\hline \multicolumn{2}{|c||}{ Input value } & Output value \\
\hline \hline Error & Error ratio & Possibility of degradation \\
\hline \hline \multirow{4}{*}{ Zero } & Zero & Zero \\
\cline { 2 - 3 } & Small & Zero \\
\cline { 2 - 3 } & Medium & Zero \\
\cline { 2 - 3 } & Big & Small \\
\hline \multirow{4}{*}{ Small } & Zero & Small \\
\cline { 2 - 3 } & Small & Small \\
\cline { 2 - 3 } & Medium & Medium \\
\cline { 2 - 3 } & Big & Medium \\
\hline \multirow{4}{*}{ Medium } & Zero & Medium \\
\cline { 2 - 3 } & Small & Medium \\
\cline { 2 - 3 } & Medium & Medium \\
\cline { 2 - 3 } & Big & Big \\
\hline \multirow{4}{*}{ Big } & Zero & Medium \\
\cline { 2 - 3 } & Small & Medium \\
\cline { 2 - 3 } & Medium & Medium \\
\cline { 2 - 3 } & Big & Big \\
\hline
\end{tabular}

Table 6. Fuzzy rules

By using fuzzy reasoning, calculation time can be shorten. Position of deterioration is identified by comparing prediction errors of each story. In this research, comparing the difference of prediction error of story, the story with the biggest difference is defined as the deterioration position. 


\subsection{Observations}

In this section, numerical examination is done using sine curve and actual wind velocity. The proposed system learned those vibration responses in advance. Figure 15 shows the difference of prediction value and observed value when external force is sine curve.

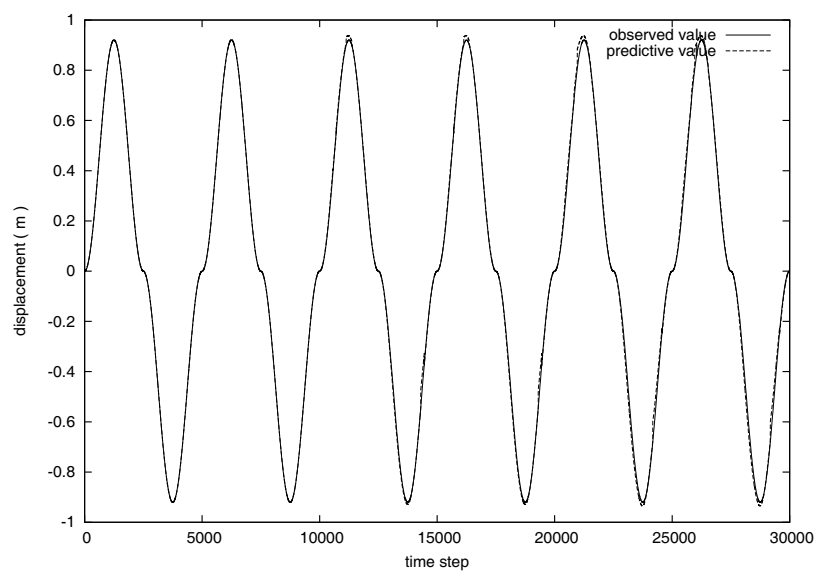

Fig. 15. Comparison of observed value and predictive value without deterioration

From Figure 15, it can be confirmed that the proposed system can identify the vibratinal characteristic by learning and predicting the vibration response. Figure 16 shows the transition of error of prediction value when the second story has deterioration.

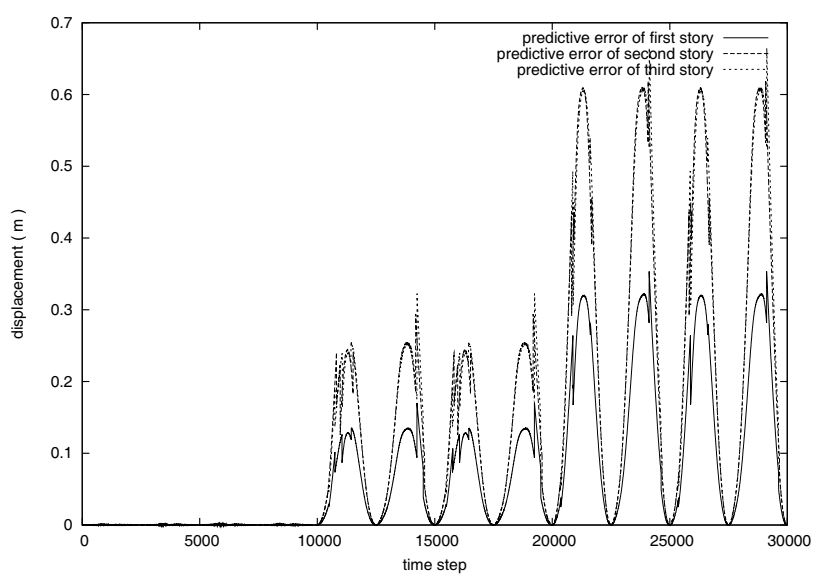

Fig. 16. Prediction error of displacement

From Figure 16, the proposed system predicts the change of stiffness and which story is deteriorated by every 10000 steps. When structural characteristic changes, the prediction error is increased. When the first story or the third story has deterioration, similar result is obtained. Figure 17 shows the output of the proposed system. 


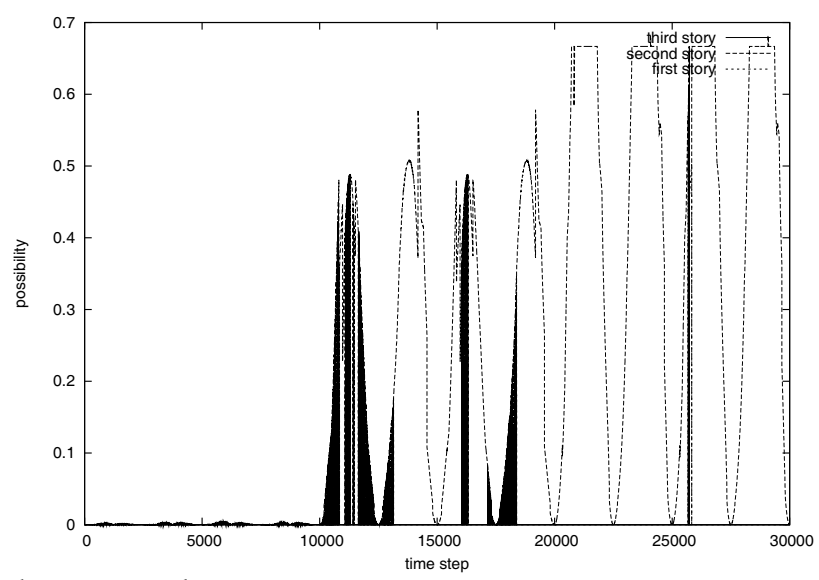

Fig. 17. Result of the proposed system

From Figure 17, the first step to 10000th step, the proposed system shows the intact state; it has no deterioration, but over 10000th step, possibility of deterioration of the second story is increased. The proposed system can identify the deterioration at real time.

Figure 18 shows the difference of prediction value and observed value when the external force is wind velocity.

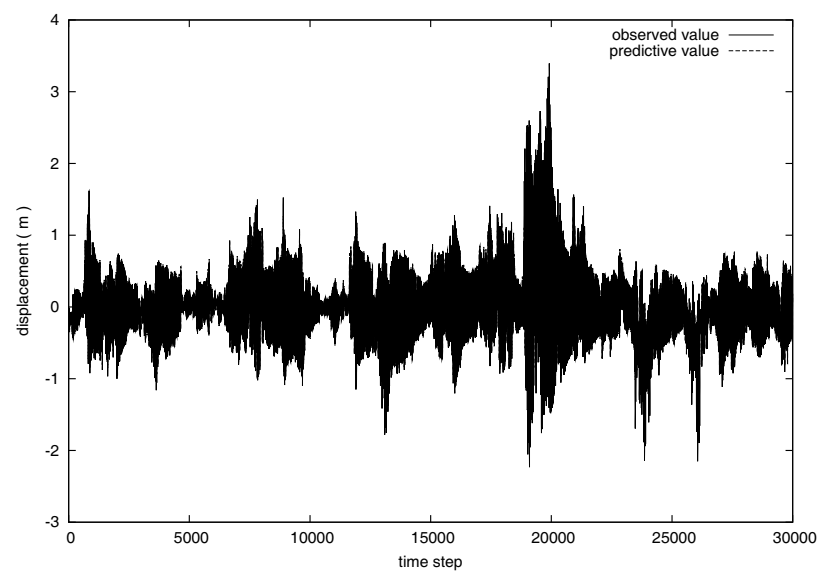

Fig. 18. Comparison of observed value and predictive value without deterioration

From Figure 18, it is confirmed that the proposed system can identify the vibrational characteristic by learning and predicting the vibration response. Figure 20 shows the transition of error of prediction value when the third story has deterioration. 


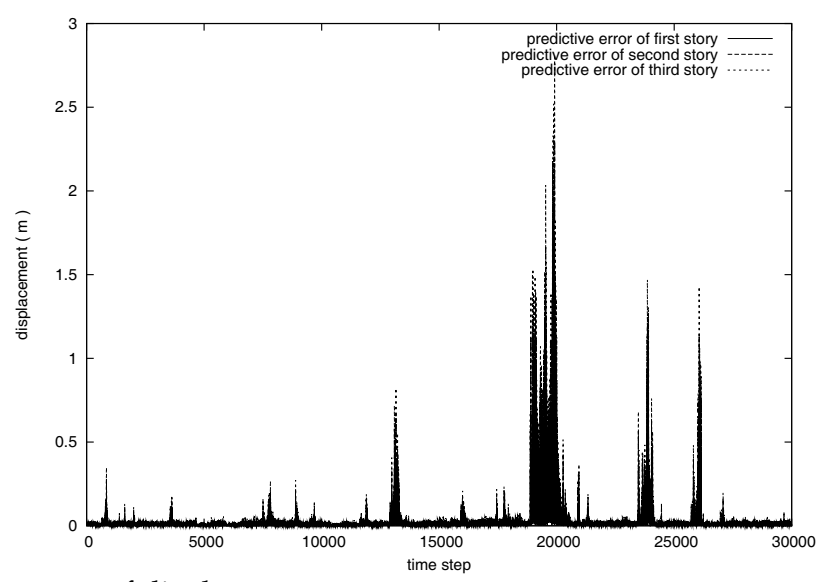

Fig. 19. Prediction error of displacement

At the simulation, stiffness is deteriorated $10 \%$ at every 10000 step. From Figure 19, the prediction error is increased by deterioration occurs. Figure 19 shows the output of the proposed system.

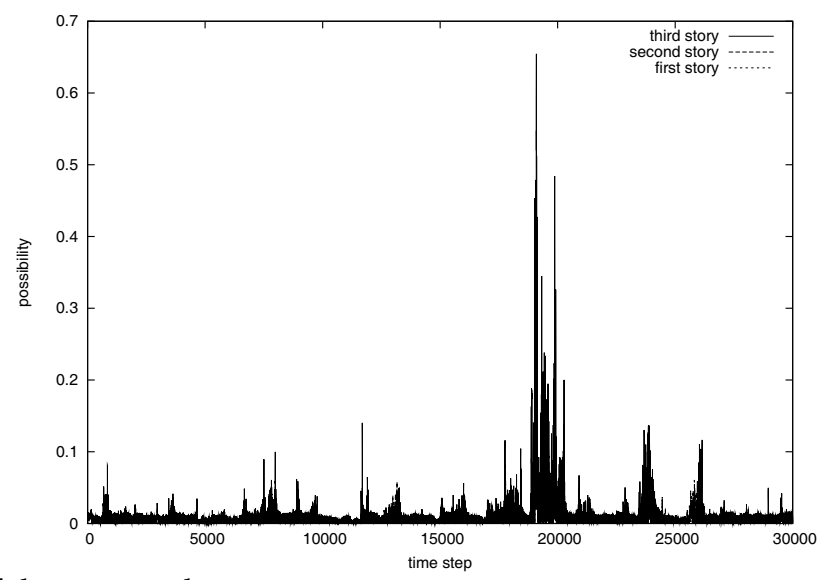

Fig. 20. Result of the proposed system

From Figure 20, up to the 10000th step, although deterioration possibility is shown, it is judged there is no deterioration because possibility is very small. After the 10000th step, although deterioration occurs, output of the proposed system hardly changes. It is due to the fact that the displacement is very small. It can be confirmed that when displacement becomes big around 20000step, output value of the proposed system becomes high. The proposed system shows the third story has deterioration. 


\section{Damage Assessment of Reinforced Concrete Bridge Decks Using AdaBoost}

In order to establish a rational maintenance program for bridge structures, it is necessary to collect enough data about the material and structural characteristics and to evaluate the structural damage of existing bridges in a quantitative manner. However, it is often seen to lose the drawings or not to record the design specification applied. Moreover, it is difficult to avoid the subjectivity of inspectors when visual data are used for the evaluation of damage or deterioration. In this section, an attempt is made to develop a new system that can evaluate the damage condition of existing structures by using the visual information given by digital photos. The proposed system is based upon such new technologies as image processing, photo-grammetry, pattern recognition, and artificial intelligence. The damage of Reinforced Concrete (RC) bridge decks is evaluated with the aid of digital photos and pattern recognition. Using the proposed system, it is possible to automatically evaluate the damage degree of RC bridge decks and therefore avoid the subjectivity of inspectors. Several numerical examples are presented to demonstrate the applicability of the proposed system.

\subsection{Damage Evaluation of RC Deck by Pattern Recognition}

In this study, the damage of Reinforced Concrete (RC) bridge decks is evaluated with the aid of digital photos and pattern recognition[9][10]. In general, the procedure for extracting the characteristics of cracks showing up on concrete decks through digital images and the classification of the damage level based on the characteristics are used in the typical pattern recognition system.

To obtain the test material, digital images of concrete decks taken by a digital camera are used. If input data that can be acquired in low resolution by using a common digital camera is used, the costs for the assessment of integrity can be reduced and input data can be acquired easily. The total number of digital images is 47 and each image is scanned with the resolution of 360 pixels per inch in both directions. In this resolution, each image is normalized to a $768 \times 480$ pixel rectangle and converted to the grayscale image. The digital images used in this study are obtained by marking the cracks with white chalk. The damage levels for all digital images are classified into three categories by an expert.

In this study, characteristics are extracted based on such four criteria as continuity, concentration, directionality (unidirectional or bi-directional), and types (hexagonal or linear) of cracks. The crack pattern of thin lines can be considered a set of directional linear elements and hence characteristic extraction by the projection histogram would be effective. Because the characteristics of projection histogram of a crack pattern provide information on the positions and the quantities of cracks, they can be used as the quantitative characteristics representing the continuity and the concentration of cracks, for the classification of crack patterns. The histograms projected on two directions are computed for extracting a crack pattern; one is the horizontal direction and the other is the vertical direction. The projection histograms are data structures used to count the number of crack pixels when the image is projected on the vertical and horizontal axes. 


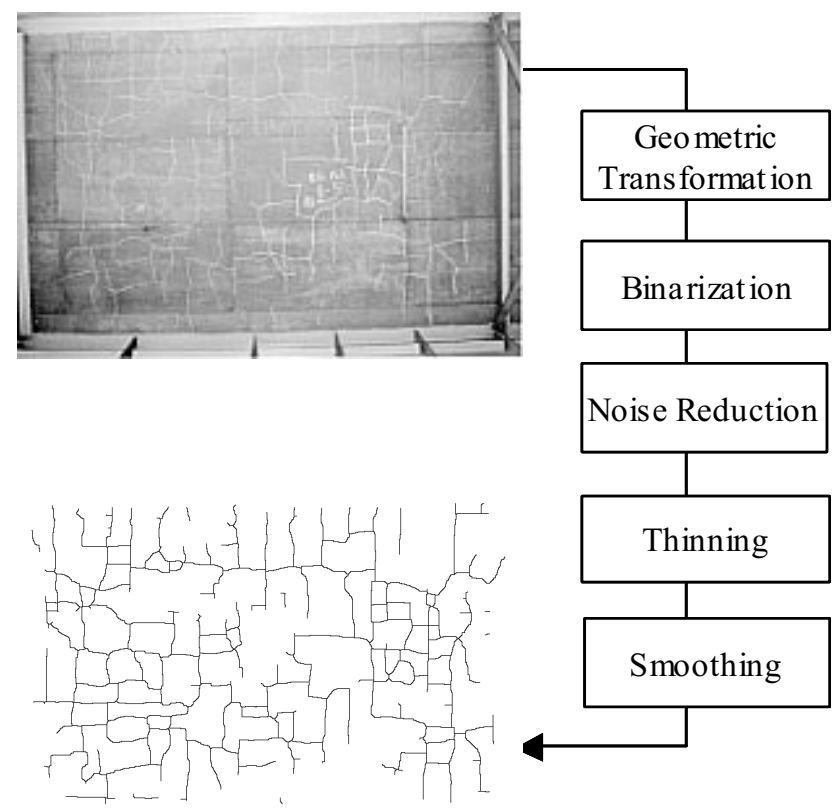

Crack Pattern

Fig. 21. Procedure of image pre-processing

The characteristic values in each dimension are the number of crack pixels in row for the horizontal histogram, in column for the vertical histogram, and are the quantum numbers in accordance with the dimensionality of characteristics vectors. Figure 22 shows an example of horizontal and vertical projection histograms extracted from a crack pattern.

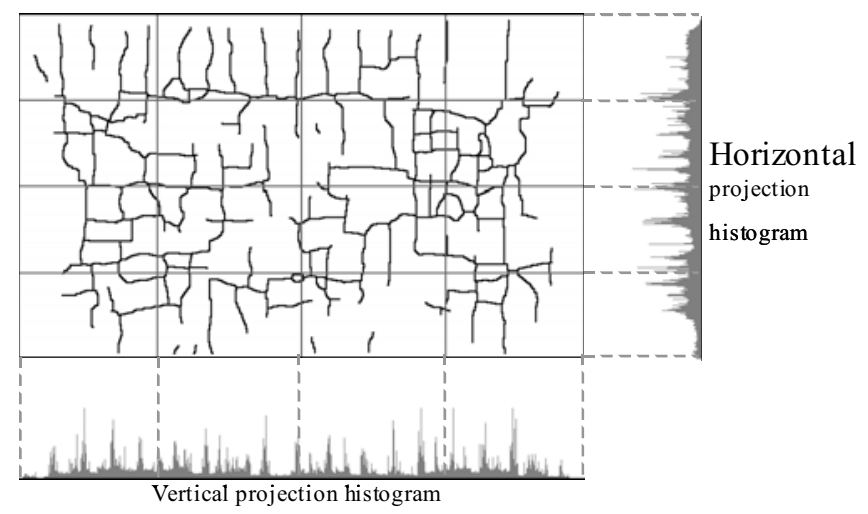

Fig. 22. Example of horizontal/vertical projection histograms extracted from a crack pattern 


\subsection{Experimental Results}

The classification of the digital images of cracks is implemented by using the AdaBoost technique. In this study, neural network is used for weak learning system. 20 images of cracks are used for learning of AdaBoost and the remaining 32 images are used for evaluating the classification results. This implementation is repeated many times changing the learning data every times. In this learning stage, three damage levels judged by an expert for each image are used as the teaching signal. Also, the teaching data includes the same number of each damage levels data. In this numerical example, the effectiveness is evaluated by the recognition rate of non-learning data and by comparing with the results of neural network. The learning parameters of neural network using back propagation and AdaBoost are shown in Table 7 and Table 8.

\begin{tabular}{|l|l|}
\hline Number of layer & 3 \\
\hline Number of unit of input layer & 512 \\
\hline Number of unit of hidden layer & 64 \\
\hline Number of unit of output layer & 3 \\
\hline Learning time & 30000 \\
\hline Learning factor & 0.3 \\
\hline Inertia factor & 0.9 inertia \\
\hline
\end{tabular}

Table 7. Leaning parameters of neural network

\begin{tabular}{|l|l|}
\hline Number of round & 30 \\
\hline Learning Time of neural network & 1000 \\
\hline
\end{tabular}

Table 8. Leaning parameters of AdaBoost

The parameters of neural network used for the weak learning system of AdaBoost are the same as Table 7. The learning parameters are set up as the same for neural network and AdaBoost so as to compare their performances. Table 9 shows the classification results with the distribution of directionality. This result is the average of all trials.

\begin{tabular}{|l|l|l|l|l|}
\hline \multirow{2}{*}{ Method } & \multicolumn{4}{|l|}{ Recognition accuracy (\%) } \\
\cline { 2 - 5 } & Rank A & Rank B & Rank C & Total \\
\hline $\begin{array}{l}\text { Neural } \\
\text { network }\end{array}$ & 60.0 & 38.5 & 87.5 & 68.1 \\
\hline AdaBoost & 90.0 & 92.3 & 95.8 & 93.6 \\
\hline
\end{tabular}

Table 9. Recognition accuracy

About $70 \%$ recognition accuracy is obtained by using neural network. Especially, the recognition rate of Rank B is very low (38.5\%). It is caused by the fact that some digital images of Rank B are close to both Rank A and Rank C. Therefore, recognition rate of Rank $\mathrm{B}$ becomes low. On the other hand, by using AdaBoost, over $90 \%$ recognition accuracy is obtained and the recognition rates of all rank are over $90 \%$. Especially, the recognition rate of Rank B is improved. From this result, AdaBoost technique can recognize the complex classification problem that neural network cannot recognize. It is considered that the proposed system can classify similar digital images by using AdaBoost. 
Table 10 shows several examples of classification by neural network. The classification is performed by the biggest value of output.

\begin{tabular}{|l|l|l|l|}
\hline \multicolumn{2}{|l|}{ Output of system } & \multirow{2}{*}{ Rank } \\
\hline A & B & C & \\
\hline 0.989 & 0.011 & 0.002 & A \\
\hline 0.011 & 0.231 & 0.159 & B \\
\hline 0.032 & 0.001 & 0.993 & C \\
\hline 0.851 & 0.154 & 0.001 & A \\
\hline 0.165 & 0.005 & 0.736 & B \\
\hline 0.006 & 0.001 & 0.999 & C \\
\hline
\end{tabular}

Table 10. Classification by neural network

From Table 10, it is seen that the classification of Rank A and Rank C can be performed in a clear manner. On the other hand, Rank B cannot be definitely classified. This implies that the classification of Rank B is complex and therefore difficult by using neural network. Table 11 shows the examples of classification by AdaBoost.

\begin{tabular}{|l|l|l|l|}
\hline \multicolumn{2}{|l|}{ Output of system } & \multirow{2}{*}{ Rank } \\
\hline A & B & C & \\
\hline 0.848 & 0.575 & 0.226 & A \\
\hline 0.550 & 0.896 & 0.189 & B \\
\hline 0.152 & 0.569 & 0.899 & C \\
\hline 0.997 & 0.602 & 0.129 & A \\
\hline 0.438 & 0.675 & 0.354 & B \\
\hline 0.139 & 0.570 & 0.918 & C \\
\hline
\end{tabular}

Table 11. Classification by AdaBoost

From Table 11, by using AdaBoost, all ranks are clearly identified. From this result, it is concluded that the AdaBoost technique is efficient even for complex problems like the problem treated here.

Table 12 shows a comparison of the recognition accuracies of learning data and checking data.

\begin{tabular}{|l|l|l|}
\hline & \multicolumn{2}{|l|}{ Recognition accuracy $(\%)$} \\
\hline & Learning data & Checking data \\
\hline $\begin{array}{l}\text { Neural } \\
\text { Network }\end{array}$ & 65.0 & 68.8 \\
\hline AdaBoost & 95.0 & 93.8 \\
\hline
\end{tabular}

Table 12. Recognition accuracies by neural network and AdaBoost

From Table 12, by using AdaBoost technique the recognition accuracy is improved for both learning data and checking data, whereas neural network has no sufficient recognition accuracy even for the learning data. This implies that neural network has a possibility not to obtain the right rules for the case with complex learning data. On the other hand, AdaBoost 
technique has higher recognition accuracy for both learning data and checking data. Namely, AdaBoost technique can obtain right rules and recognize complex problems by combining several weak learning systems. From this, it can be confirmed that AdaBoost technique is quite efficient for the evaluation of damage condition of RC bridge decks.

\section{Optimal Maintenance Planning Considering Health Monitoring Information}

In order to establish a rational bridge maintenance program, it is inevitable to determine the deterioration curves of various components of bridges. However, it is quite difficult to identify appropriate deterioration curves because of various uncertainties involved in the prediction process and environment evaluation. Furthermore, it is necessary to take into account the effects of natural hazards such as earthquake, typhoon, flood, etc. Under the situation, although the existing bridges should be kept in a safety condition through inspection and maintenance works, it is not easy to maintain them in a satisfactory level, because of the financial constraints. In this study, an attempt is made to develop an optimal maintenance planning system that can account for the seismic risk. In the proposed system, an optimal maintenance scheduling is obtained with the aid of Genetic Algorithm (GA), in which the deterioration curve can be updated by using the data given from the structural health monitoring. Introducing the health monitoring information, it becomes possible to change the repair intervals or repair methods that can reduce Life-Cycle Cost (LCC) and seismic risk. The structural health monitoring is performed using fuzzy reasoning. Several numerical examples are presented to demonstrate the applicability and efficiency of the proposed method.

\subsection{Problem of Maintenance Scheduling}

The objective of bridge maintenance is to keep the bridge condition well and to lengthen its life. In order to establish an appropriate maintenance schedule for the bridge, it is very important to minimize the maintenance cost. However, there is a necessity to consider the risks of damage and failure caused by earthquakes. Then, since there is a trade-off relation between the maintenance cost and seismic risk, it is very difficult to satisfy both the objectives.

In the past researches, the trade-off has been treated by using the multi-objective optimization of the maintenance cost and seismic risk. However, the multi-objective optimization methods provide a lot of solutions so that it is not easy to choose a solution among the solution candidates. Also, there is a big problem at the prediction of the deterioration rate. Since the deterioration has uncertainties, the accuracy of the prediction is low. The low accuracy of the prediction may cause the increase of seismic risk. Namely, it is important to specify an appropriate deterioration rate.

\subsection{Maintenance Scheduling Considering Seismic Risk}

LCC of bridge structures consists of initial construction cost, maintenance cost, and failure cost (renewal cost, user cost, social and environmental costs and so on). In usual, LCC analysis considers the damage and deterioration of materials and structures. However, in the region that often suffers from natural hazards such as typhoons and earthquakes, it is necessary to account for the effects of such natural hazards. 
Based on the seismic risk analysis, LCC is evaluated focusing on the effects of earthquakes that are major natural disasters in Japan. At first, LCC analysis is formulated to consider the social and economical effects due to the collapse of structures occurred by the earthquake as well as the minimization of maintenance cost. The loss by the collapse of structures due to the earthquake can be defined in terms of an expected cost and introduced into the calculation of LCC. A stochastic model of structural response was proposed, which accounts for the variation due to the uncertain characteristics of earthquake [11]. Then, the probability of failure due to the earthquake excitation is calculated based on the reliability theory. Furthermore, LCC evaluations are performed not only for a single bridge but also many bridges forming road networks [12][13].

In the past researches [14][15], both the maintenance cost and seismic risk were treated by using the multi-objective optimization technique. However, it is not easy to select an appropriate maintenance plan among a lot of candidates that the multi-objective optimization provides as a set of Pareto solutions.

As mentioned before, there is such another big problem that the prediction of the deterioration curve essentially involves various uncertainties. This implies that the accuracy of the prediction is low and insufficient, which may cause the increase of the seismic risk.

\subsection{Maintenance Planning System Considering Seismic Risk}

In this section, an attempt is made to develop a maintenance scheduling system that can consider the maintenance cost and seismic risk at the same time to achieve a rational maintenance program by using GA.

\subsubsection{Objective function}

In this research, the objective function is the maintenance cost.

\subsubsection{Sub-objectives}

There are a lot of efficient solutions when optimizing only the life cycle cost. Although the maintenance cost is equal, the characteristics of the maintenance schedule are different from each other. Therefore, when the maintenance cost is equal, the seismic risk is considered as a sub-objective. The seismic risk is defined in the following:

$$
\text { SeismicRisk }=P_{d} \times P_{h} \times C_{d}
$$

where $P_{d}$ is the probability of seismic damage occurrence, $P_{h}$ is the earthquake occurrence probability and $C_{d}$ is the seismic loss.

\subsubsection{Introduction of health monitoring}

In this research, it is assumed that the health monitoring system can recognize the deterioration rate with accuracy. When the prediction error is large, the maintenance schedule is updated by GA. By updating the schedule, the seismic risk can be reduced. The flow of the proposed system is shown in Figure 23 and the updating process of the maintenance schedule is shown in Figure 24. By introducing the health monitoring, it is possible to reduce the maintenance cost and seismic risk. 


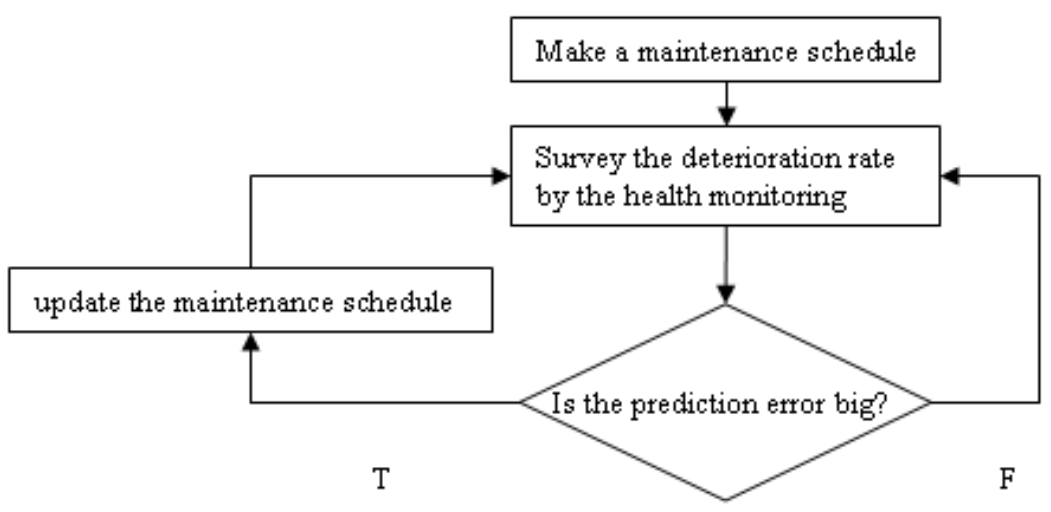

Fig. 23. Flow of the proposed system
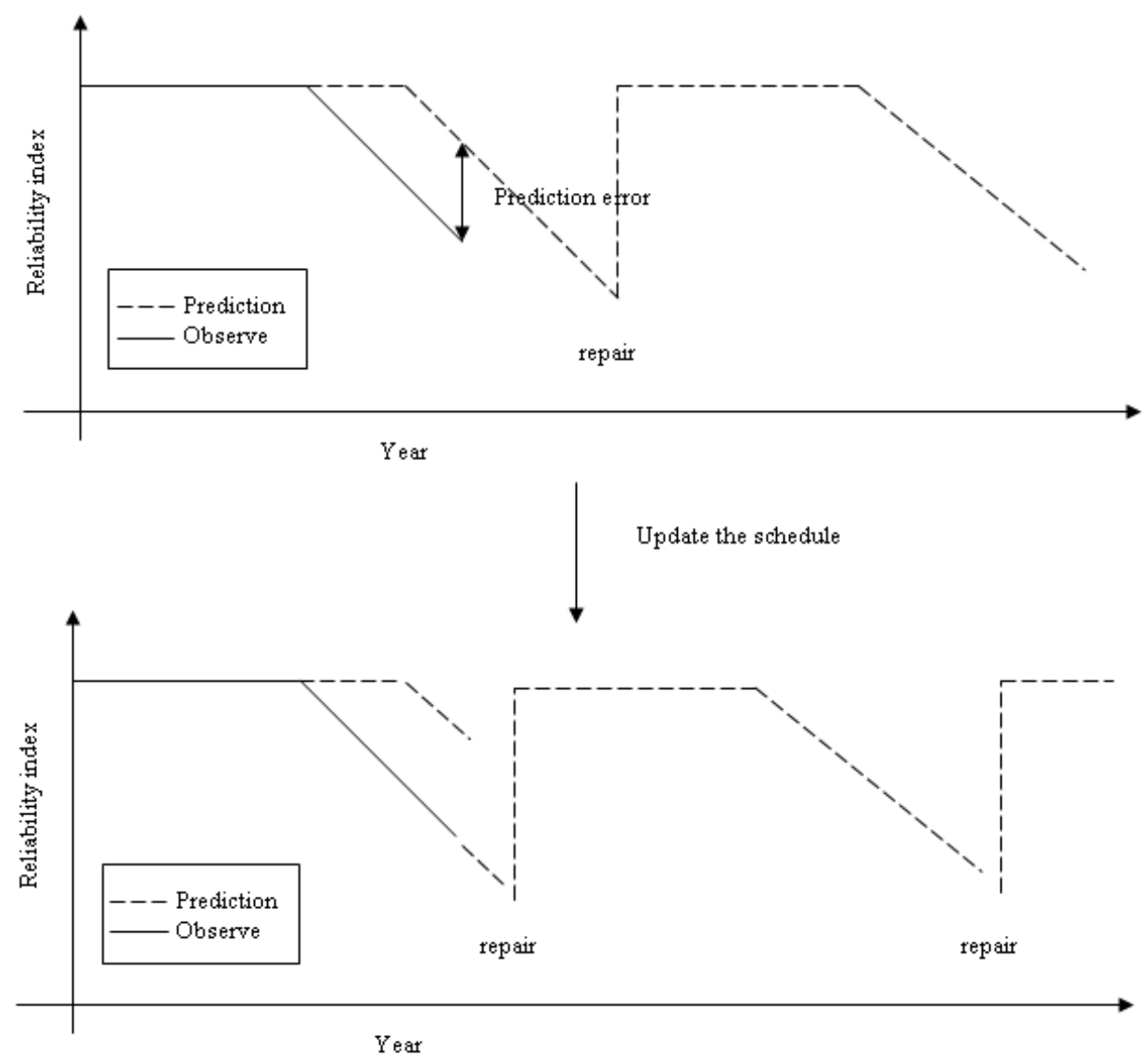

Fig. 24. Updating process of maintenance schedule 
When the prediction error is large, the deterioration curve can be updated by using the data obtained from the health monitoring system and the optimal maintenance schedule is searched by GA based on the updated deterioration curve.

\subsubsection{Uncertainty of deterioration}

Figure 25 shows the deterioration curve of a member under the severe environment. The deterioration accelerates after 27 years. It is assumed that the preventive effects for corrosion lose after 27 years, the required safety level is prescribed as 0.8 , and the service life is 100 years.

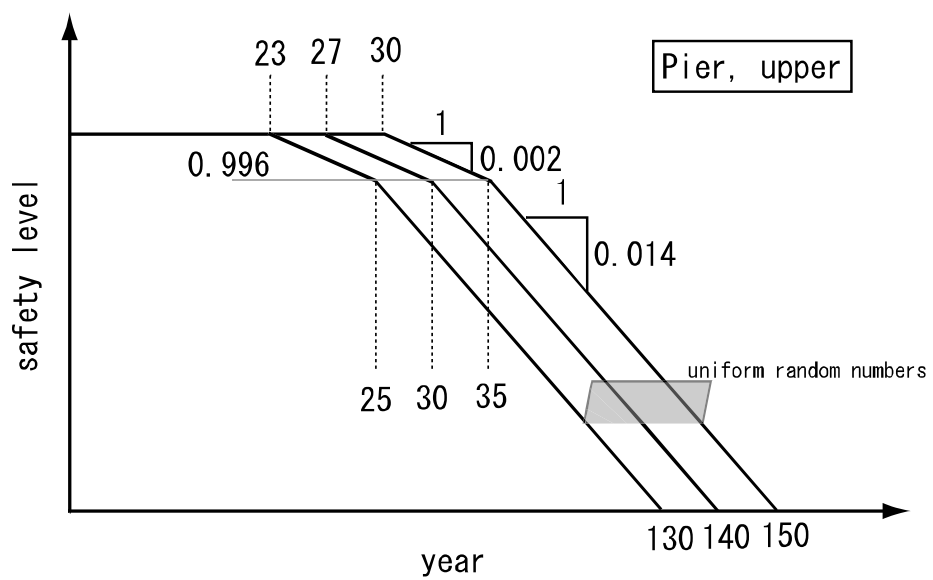

Fig. 25. Deterioration curve of RC member

\subsection{Numerical Example}

A numerical examination for a bridge is presented. The uncertainty of the deterioration curve is expressed by the normal distribution. In this numerical example, it is assumed that the health monitoring system can update the deterioration curve every year. The result of the proposed system with health monitoring system is compared with the systems without health monitoring. The simulation is done 100 times and the result is shown in Table 13.

\begin{tabular}{lll}
\hline Seismic Risk & $\begin{array}{c}\text { Monitoring } \\
\text { Without }\end{array}$ & With \\
\hline Max & 180477 (Yen) & 155767 (Yen) \\
Min & 169817 (Yen) & 155272 (Yen) \\
Average & 176872 (Yen) & 155469 (Yen) \\
\hline
\end{tabular}

Table 13. Seismic risk

Table 13 shows that the proposed method with health monitoring can provide less seismic risk than that without health monitoring, by using Equation (25). In this table, Max, Min and Average mean the maximum seismic risk, the minimum seismic risk and the average seismic risk in 100 times simulation. This result shows the proposed system can adapt to the 
uncertainties of the deterioration by introducing the health monitoring system. Since the maintenance cost of the proposed system is almost the same, the effectiveness of the proposed system is verified.

\section{References}

[1] Sugimoto, H. \& Katagiri, A. (1997): On Support System for Restoration Process of Disaster-Stricken Lifeline Network by GA, Vol. 43, No. 2. pp. 517-524. (in Japanese).

[2] Furuta, H. \& Nakatsu, K. (2004): Optimal Restoration Scheduling for Earthquake Disaster by Emergent Computing, Proc. of IFIP WG7.5 Working Conference on Reliability and Optimization of Structural Systems, Kobe, Japan.

[3] Tamaki, H., Arai, T. \& Abe, S. (1999): A Genetic Algorithm Approach to Optimization Problems with Uncertainties", Institute of Systems, Control and Information Engineers Journal, Vol. 12, No. 5, pp. 297-303. (in Japanese).

[4] Frangopol, D. M. \& Furuta, H. (eds.), (2001). Life-Cycle Cost Analysis and Design of Civil Infrastructure Systems, ASCE, Reston Virginia.

[5] Ito H., Takahashi Y., Furuta, H., \& Kameda, T. (2002): An Optimal Maintenance Planning for Many Concrete Bridges Based on Life-Cycle Cost. Proc. of IABMAS, Barcelona, Spain, CD-ROM.

[6] Furuta, H., Kameda, T. \& Frangopol, D. M. (2004): Balance of Structural Performance Measures, Proc. of Structures Congress, Nashville, Tennessee, ASCE, May, CD-ROM.

[7] MLTI. (2001). Cost Estimation Standards for Civil Constructions, Ministry of Land, Transportation and Infrastructure, Japan.

[8] Furuta, H. \& Sugimoto, H. (1997). Applications of Genetic Algorithm to Structural Engineering, Tokyo, Morikita Publishing (in Japanese).

[9] Yagi, N. (2000): Digital Image Processing, Ohmsha (in Japanese)

[10] Seul, M., O'Gorman, L. \& Sammon, M. (2001): Practical Algorithms for Image Analysis, Cambridge University Press

[11] Furuta, H., Koyama, K., Dogaki, M., \& Frangopol, D. M. (2004): Seismic Risk Evaluation and Life-Cycle cost Analysis of Bridge Structures in Japan, Proc. of 2nd ASRANeT Colloquium, Barcelona, Spain.

[12] Furuta, H., Kataoka, H., Dogaki, M. \& Frangopol, D. M. (2005): Effects of Seismic Risk on Life-Cycle Cost Analysis for Bridge Maintenance, Proc. of 4th International Conference on Current and Future Trends in Bridge, Construction and Maintenance, Kuala Lumpur, Malaysia.

[13] Furuta, H., Koyama, K., Oi, M. \& Sugimoto, H. (2006): Life-Cycle Cost Evaluation of Multiple Bridges in Road Network Considering Seismic Risk, Proc. of 6th International Bridge Engineering Conference, Boston, USA.

[14] Furuta, H., Koyama, \& Oi, M. (2005): Life-cycle Analysis of Bridge Structures Considering Maintenance Cost and Seismic Risk, Proc. of IFIP WG7.5 Working Conference on Structural Reliability and Optimization, Aalborg, Denmark.

[15] Furuta, H., Nakatsu, K. \& Frangopol, D. M. (2005): Optimal Restoration Scheduling for Earthquake Disaster Using Life-Cycle Cost, Proc. of 4th International Workshop on Life-Cycle Coast Analysis and Design of Civil Infrastructure Systems, Coco Beach, USA. 


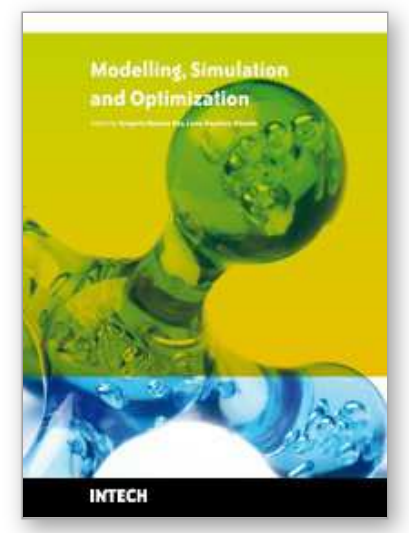

\author{
Modelling Simulation and Optimization \\ Edited by Gregorio Romero Rey and Luisa Martinez Muneta
}

ISBN 978-953-307-048-3

Hard cover, 708 pages

Publisher InTech

Published online 01, February, 2010

Published in print edition February, 2010

Computer-Aided Design and system analysis aim to find mathematical models that allow emulating the behaviour of components and facilities. The high competitiveness in industry, the little time available for product development and the high cost in terms of time and money of producing the initial prototypes means that the computer-aided design and analysis of products are taking on major importance. On the other hand, in most areas of engineering the components of a system are interconnected and belong to different domains of physics (mechanics, electrics, hydraulics, thermal...). When developing a complete multidisciplinary system, it needs to integrate a design procedure to ensure that it will be successfully achieved. Engineering systems require an analysis of their dynamic behaviour (evolution over time or path of their different variables). The purpose of modelling and simulating dynamic systems is to generate a set of algebraic and differential equations or a mathematical model. In order to perform rapid product optimisation iterations, the models must be formulated and evaluated in the most efficient way. Automated environments contribute to this. One of the pioneers of simulation technology in medicine defines simulation as a technique, not a technology, that replaces real experiences with guided experiences reproducing important aspects of the real world in a fully interactive fashion [iii]. In the following chapters the reader will be introduced to the world of simulation in topics of current interest such as medicine, military purposes and their use in industry for diverse applications that range from the use of networks to combining thermal, chemical or electrical aspects, among others. We hope that after reading the different sections of this book we will have succeeded in bringing across what the scientific community is doing in the field of simulation and that it will be to your interest and liking. Lastly, we would like to thank all the authors for their excellent contributions in the different areas of simulation.

\title{
How to reference
}

In order to correctly reference this scholarly work, feel free to copy and paste the following:

Hitoshi Furuta, Koichiro Nakatsu and Hiroshi Hattori (2010). Applications of Soft Computing in Engineering Problems, Modelling Simulation and Optimization, Gregorio Romero Rey and Luisa Martinez Muneta (Ed.), ISBN: 978-953-307-048-3, InTech, Available from: http://www.intechopen.com/books/modelling-simulationand-optimization/applications-of-soft-computing-in-engineering-problems

\section{INTECH}

open science | open minds

\section{InTech Europe}

University Campus STeP Ri

Slavka Krautzeka 83/A

\section{InTech China}

Unit 405, Office Block, Hotel Equatorial Shanghai

No.65, Yan An Road (West), Shanghai, 200040, China 
51000 Rijeka, Croatia

Phone: +385 (51) 770447

Fax: +385 (51) 686166

www.intechopen.com
中国上海市延安西路65号上海国际贵都大饭店办公楼405单元

Phone: +86-21-62489820

Fax: $+86-21-62489821$ 
(C) 2010 The Author(s). Licensee IntechOpen. This chapter is distributed under the terms of the Creative Commons Attribution-NonCommercial-ShareAlike-3.0 License, which permits use, distribution and reproduction for non-commercial purposes, provided the original is properly cited and derivative works building on this content are distributed under the same license. 